\title{
Laser-induced Structural Modifications inside Glass Using a Femtosecond Laser and a $\mathrm{CO}_{2}$ Laser
}

\author{
Takuto ASADA ${ }^{* 1}$, Takayuki TAMAKI ${ }^{* 1}$, Masaya NAKAZUMI ${ }^{* 1}$, Etsuji OHMURA $^{* 2}$, and Kazuyoshi ITOH ${ }^{* 3}$ \\ ${ }^{* 1}$ Department of Control Engineering, Nara National College of Technology, \\ Institute of National Colleges of Technology, Japan \\ 22 Yata-cho, Yamatokoriyama, Nara 639-1080, Japan \\ a0723@stdmail.nara-k.ac.jp \\ ${ }^{* 2}$ Management of Industry and Technology, Graduate School of Engineering, Osaka University \\ 2-1Yamadaoka, Suita, Osaka 565-0871, Japan \\ *3 Science Technology Entrepreneurship Laboratory (e-square), Graduate School of Engineering, Osaka University \\ 2-1Yamadaoka, Suita, Osaka 565-0871, Japan
}

\begin{abstract}
In this paper, we present laser-induced structural modifications inside BK7 glass (OHARA, S-BSL7) using a femtosecond laser and a $\mathrm{CO}_{2}$ laser system. A femtosecond laser has extremely short pulse duration (less than $10^{-12} \mathrm{sec}$ ) and high peak power. When an intense femtosecond laser pulse irradiates inside transparent material, the nonlinear absorption phenomenon generates around the focal spot, and leads to a localized structural modification in the material. Also $\mathrm{CO}_{2}$ laser can generate the thermal effect around laser-irradiated area. By combining the nonlinear absorption and thermal effect, we develop novel femtosecond laser microprocessing using thermal effect. We found the 2-wavelengths laser microprocessing (TLAM) has the effect of displacing the focal point of femtosecond laser pulse with the increase of the $\mathrm{CO}_{2}$ laser power. Moreover the TLAM increases heat-affected zone in the unit time than that using single femtosecond laser microprocessing (SFLAM). The processed diameter using TLAM is approximately twice the size of the diameter using SFLAM. The increase of the diameter of structural modifications expands the possibilities of the application to the three dimensional microfabrication inside the transparent material.
\end{abstract}

DOI: $10.2961 /$ jlmn.2014.02.0017

Keywords: 2-wavelengths laser microprocessing system, femtosecond laser, $\mathrm{CO}_{2}$ laser, structural modification, surface ablation, internal processing, glass

\section{Introduction}

Ultrashort laser pulse has great potential for the threedimensional (3D) microprocessing in transparent material because the pulse has extremely short pulse duration (less than $10^{-12} \mathrm{sec}$ ) and high peak power. When ultrashort laser pulses irradiate inside transparent material, the laser pulses can become high enough to cause localized permanent structural modifications in the material $[1,2]$. Papers on the 3D microfabrication inside the transparent material with the structural modification by single femtosecond laser system have been reported. For example, the 3D optical devices such as waveguides [3, 4], couplers [5], lenses [6], and gratings $[7,8,9,10]$ have been fabricated inside various glass materials.

The size of structural modifications in these devices depends on the pulse energy of femtosecond laser. If the diameter of structural modifications is larger than the ordinary size, the possibilities of the application to the 3D microfabrication inside the transparent material will expand (e.g. reduction of processing time). However, in order to obtain large structural modifications, high-energy femtosecond laser is generally required. For example we investigated the relationship between diameter of structural modifications inside BK7 glass and the pulse energy of femtosecond laser by focusing femtosecond laser pulses (wavelength: $1.064 \mu \mathrm{m}$, pulse duration: $400 \mathrm{fs}$, and repetition rate: $1 \mathrm{MHz}$ ) with a $0.40-\mathrm{NA}$ (numerical aperture) objective lens. Figure 1 shows the obtained relationship and the primary curve that is calculated from the diameter of pro- cessed structural modifications. From the primary curve the expected diameter of structural modifications with more than $1.4 \mu \mathrm{J}$ in pulse energy of femtosecond laser is estimated and denoted with a dotted line in Fig.1. For example, in order to form the $100 \mu \mathrm{m}$ diameter of structural modifications, more than $2 \mu \mathrm{J}$ in pulse energy of femtosecond laser pulse energy will be required. However, a high-energy femtosecond laser generally needs space and cost for the installation and operation.

In this paper to create large diameter of structural modification using low-energy femtosecond laser (for example up to $1.4 \mu \mathrm{J}$ ), we propose the 2 -wavelengths laser microprocessing (TLAM), which consists of $\mathrm{CO}_{2}$ laser and femtosecond laser. The $\mathrm{CO}_{2}$ laser beam has high-power continuous or pulse wave in the infrared region. Therefore the thermal effects will occur in the laser-irradiated area

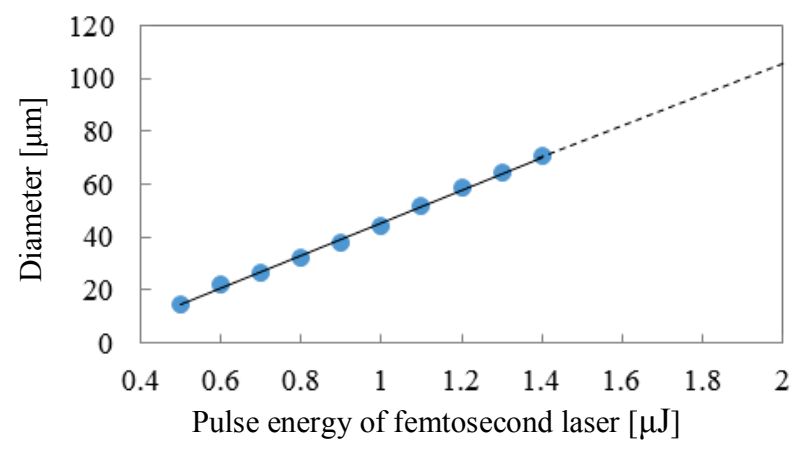

Fig. 1 Relationship between diameter of structural modifications inside BK7 glass and pulse energy of femtosecond laser. 
$[11,12,13,14]$. By use of the thermal effect with $\mathrm{CO}_{2}$ laser and the nonlinear phenomenon with femtosecond laser, TLAM is developed.

\section{Principle of TLAM}

\subsection{The processed amount inside glass using TLAM or FSLAM}

Figure 2 shows the schematic diagram of single femtosecond laser microprocessing (FSLAM) and the 2wavelengths laser microprocessing (TLAM). In order to investigate the effect of FSLAM and TLAM, femtosecond laser pulses without/with $\mathrm{CO}_{2}$ laser beam irradiate inside BK7 glass (200 $\mu \mathrm{m}$ depth from glass surface). When femtosecond laser pulses irradiate transparent material, the laser-induced structural modifications are created around focal spot through the nonlinear and avalanche ionization phenomenon. By providing the energy of $\mathrm{CO}_{2}$ laser beam to the material with the irradiation of femtosecond laser, we cosider that the avalanche ionization is likely to be occur [15]. Therefore the diameters of the laser-induced structural modifications using TLAM will be larger than that using FSLAM. Thus the heataffected zone in the unit time using TLAM increases than that using SFLAM.

\subsection{Displacement of focal position}

When femtosecond laser pulses irradiate on the surface of transparent material, the pulse causes ablation around the focal spot. However, when $\mathrm{CO}_{2}$ laser beam and femtosecond laser pulses irradiate on the surface of the material simultaneously, internal processing is realized because the movement of the focal point occurs. Although the mechanism about the movement is still under investigation, we consider that the factor is probably thermal lens effect and/or thermal expansion of the glass.

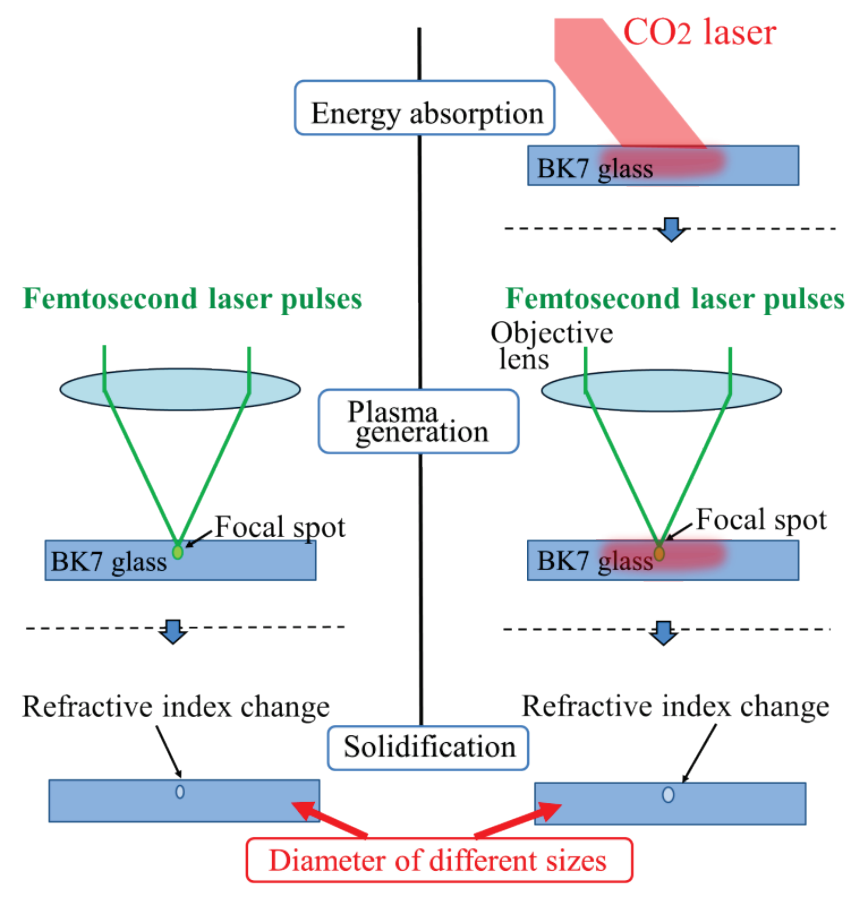

(a) FSLAM

(b) TLAM

Fig. 2 The schematic diagram of FSLAM and TLAM.

\section{Experimental setup}

Figure 3 shows an optical setup for the 2-wavelengths laser microprocessing (TLAM) that consists of femtosecond fiber laser and $\mathrm{CO}_{2}$ laser systems. A femtosecond fiber laser system (Fianium, FP1060S-PP-D) generates $1.064 \mu \mathrm{m}$, $400 \mathrm{fs}$, and $1 \mathrm{MHz}$ ultrafast laser pulses with pulse energy up to $2 \mu \mathrm{J}$ (immediately after output of laser system). It should be noted that the $1 \mathrm{MHz}$-repetition-rate leads to the heat accumulation in BK7 glass (OHARA, S-BSL7, specifications of the glass are shown in Table 2 [15]). Also $\mathrm{CO}_{2}$ laser system (Kantum Electronics, H48-1-28SW) generates pulsed laser beam with a repetition rate of $5 \mathrm{kHz}$ and a wavelength of $10.6 \mu \mathrm{m} . \mathrm{CO}_{2}$ laser beam was irradiated to glass for 5 seconds in order to provide a sufficient heat effect. Next femtosecond laser pulses were irradiated simultaneously behind the $\mathrm{CO}_{2}$ laser irradiation. The pulse energy of femtosecond laser was controlled by rotating a half-wave plate in front of a Glan-laser polarizer. The output power was controlled by the duty ratio of electrical pulse in the $\mathrm{CO}_{2}$ laser driver. Table 3 shows the beam size of the $\mathrm{CO}_{2}$ laser beam with different laser output. The sample to be processed was fixed a computer-controlled threedimensional stage. Femtosecond laser pulses were focused on the glass surface or inside glass with a $20 \times$ objective lens (Olympus LMPlan 20×IR) of a 0.40 numerical aperture. By translating the glass sample perpendicular to the optical axis of femtosecond laser pulses with a distance of $1 \mathrm{~mm}$, the linear structural modification regions were created. The laser-induced modification region were observed in the $x y$-plane and in the $x z$-plane by optical transmission microscopes with white-light illumination.

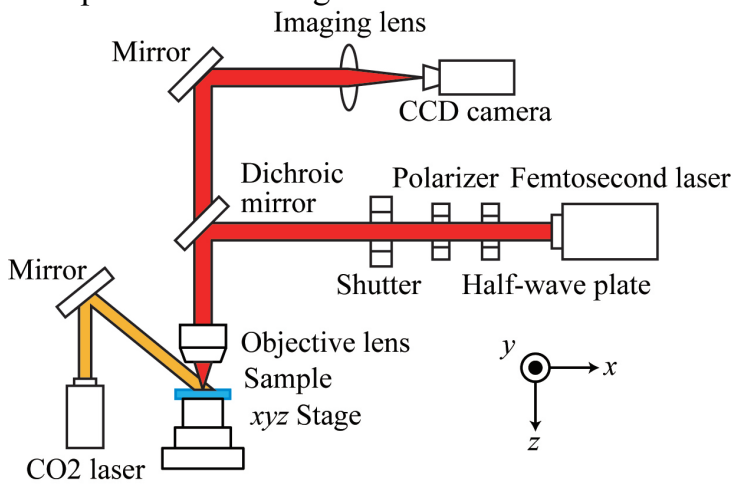

Fig. 3 Schematic diagram of optical setup used for TLAM using femtosecond laser and $\mathrm{CO}_{2}$ laser systems. $\mathrm{CCD}$ : chargecoupled device camera.

Table 1 Characteristics of S-BSL 7 glass [16].

\begin{tabular}{cc}
\hline Dimension $[\mathrm{mm}]$ & $5 \times 30$ \\
\hline Thickness $[\mathrm{mm}]$ & 0.67 \\
\hline Refractive index $[-]$ & 1.51 \\
\hline Transition point $\left[{ }^{\circ} \mathrm{C}\right]$ & 576 \\
\hline Softening point $\left[{ }^{\circ} \mathrm{C}\right]$ & 718 \\
\hline Coefficient of thermal conductivity & 1.13 \\
\hline $\mathrm{W} / \mathrm{m} \cdot \mathrm{K}]$ & 532 \\
\hline Strain point $\left[{ }^{\circ} \mathrm{C}\right]$ & 563 \\
\hline Annealing point $\left[{ }^{\circ} \mathrm{C}\right]$ & 625 \\
\hline Deformation point $\left[{ }^{\circ} \mathrm{C}\right]$ & $-30 \sim 70\left[{ }^{\circ} \mathrm{C}\right]$ \\
\hline Coefficient of linear expansion & \\
\hline
\end{tabular}


$\left[10^{-7} /{ }^{\circ} \mathrm{C}\right]$

$100 \sim 300\left[{ }^{\circ} \mathrm{C}\right]$

86

Table 2 The beam size of the $\mathrm{CO}_{2}$ laser beam.

\begin{tabular}{cc}
\hline $\mathrm{CO}_{2}$ laser output [W] & $\begin{array}{c}\text { The laser beam diameter } \\
{[\mathrm{mm}]}\end{array}$ \\
\hline 2 & 8.5 \\
\hline 3 & 9.5 \\
\hline 4 & 10.0 \\
\hline
\end{tabular}

\section{Results and discussions}

\subsection{Surface ablation}

4.1.1 Morphology dependence on the thermal effect of $\mathrm{CO}_{2}$ laser beam

In order to clarify the influence of the thermal effect of the $\mathrm{CO}_{2}$ laser beam, we demonstrated laser microprocessing of BK7 glass by using (i) single femtosecond laser microprocessing (FSLAM) or (ii) the 2wavelengths laser microprocessing (TLAM). The pulse energy of femtosecond laser was set to $0.6 \mu \mathrm{J}$. The output power of $\mathrm{CO}_{2}$ laser was set to $3 \mathrm{~W}$. Figures 4 (a) and (b) show optical microscope images of laser-induced structural modification in BK7 glass using FSLAM and TLAM, respectively. From Figs. 4(a) and (b), the laserinduced structural modification is surface ablation for FSLAM, and that is refractive-index change inside glass for TLAM.

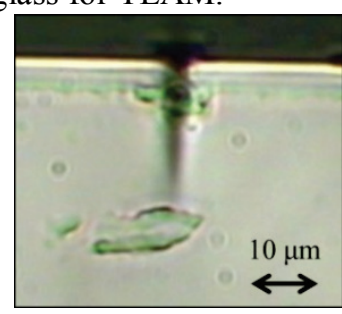

(a) FSLAM

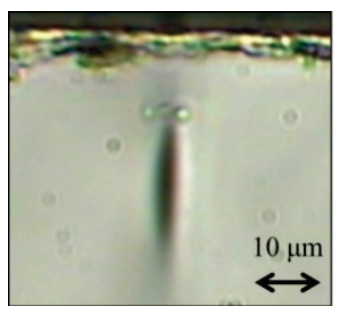

(b) TLAM
Fig. 4 Optical microscope images of laser-induced structural modification in BK7 glass using (a) FSLAM and (b) TLAM, respectively.

\subsubsection{Morphology dependence on output power of $\mathrm{CO}_{2}$ laser beam and/or energy of femtosecond laser pulses}

We investigated the laser-induced morphology (surface ablation, internal processing, and intermediate state (i.e. surface ablation region changed to internal processing during laser processing)) in BK7 glass by changing the output power of $\mathrm{CO}_{2}$ laser beam and/or energy of femtosecond laser pulses. Figure 5 shows the examples of surface ablation and internal processing in the laserinduced morphology. The pulse energy of femtosecond laser was changed to $0.60 \mu \mathrm{J}, 0.65 \mu \mathrm{J}, 0.70 \mu \mathrm{J}$, and 0.75 $\mu \mathrm{J}$. The output power of $\mathrm{CO}_{2}$ laser beam was also changed to $1 \mathrm{~W}, 2 \mathrm{~W}, 3 \mathrm{~W}$, and $4 \mathrm{~W}$. Table 4 shows the laser-induced morphology in each condition. In Table 4, open circle ( $\circ$ ), cross mark $(\times)$, and open square $(\square)$ show internal processing, surface ablation, and intermediate state, respectively. From Table 4 we confirm that the laser-induced morphologies are internal processing or intermediate state with more than $2 \mathrm{~W}$ output power of $\mathrm{CO}_{2}$ laser beam. Note that, with more than 5 $\mathrm{W}$ output power of $\mathrm{CO}_{2}$ laser beam, the $\mathrm{BK} 7$ glass sample physically broke. Therefore the morphology depends on the power of $\mathrm{CO}_{2}$ laser beam.

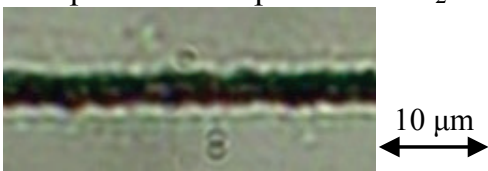

(a) Surface ablation

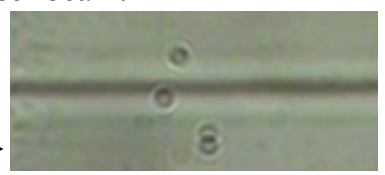

(b) Internal processing
Fig. 5 Examples of surface ablation and internal processing in the laser-induced morphology.

Table 3 The laser-induced morphology in each condition. Open circle $(\circ)$, cross mark $(\times)$, and open square $(\square)$ show internal processing, surface ablation, and intermediate state, respectively.

Energy of
$\begin{aligned} & \text { Power of } \\ & \text { femtosecond } \\ & \text { laser pulses }[\mu \mathrm{J}]\end{aligned}$

\subsubsection{Depth dependence of laser-induced morpholo- gy on output power of $\mathrm{CO}_{2}$ laser beam and/or energy of femtosecond laser pulses}

We investigated the depth of laser-induced morphology from the glass surface by changing the output power of $\mathrm{CO}_{2}$ laser beam and/or energy of femtosecond laser pulses. The pulse energy of femtosecond laser was changed to $0.60 \mu \mathrm{J}, 0.65 \mu \mathrm{J}, 0.70 \mu \mathrm{J}$, and $0.75 \mu \mathrm{J}$. The output power of $\mathrm{CO}_{2}$ laser beam was also changed to 1 $\mathrm{W}, 2 \mathrm{~W}, 3 \mathrm{~W}$, and $4 \mathrm{~W}$. Figure 6 shows the cross section of a laser-induced morphology and definition of depth in the morphology. Also Fig. 7 shows relationship between the depth of the morphology and the power of $\mathrm{CO}_{2}$ laser beam. From Fig. 7 the depth is increased with an increase in the power of $\mathrm{CO}_{2}$ laser beam. About the dependence of the depth on the energy of femtosecond laser pulses, in the region of internal processing, the depth depends on the energy of femtosecond laser pulse. In contrast, in the region of surface ablation, the depth doesn't depend on the energy.

We consider that the reason for the dependence of the depth on output power of $\mathrm{CO}_{2}$ laser beam and energy of femtosecond laser pulses is likely the thermal effect that is described in Section 2. The generation of the thermal effect is shown during laser microprocessing using TLAM (see Fig. 8). From Fig. 8 we confirm that the image during processing is blurred. The reason is likely thermal expansion due to the increase of temperature with energy absorption of $\mathrm{CO}_{2}$ laser beam into the glass although we will investigate the mechanism on the displacement of focal position in detail in the future.

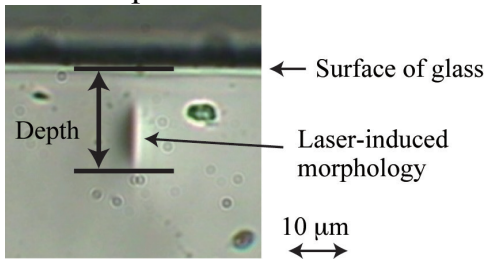


Fig. 6 Cross section of laser-induced morphology and definition of depth in the morphology.

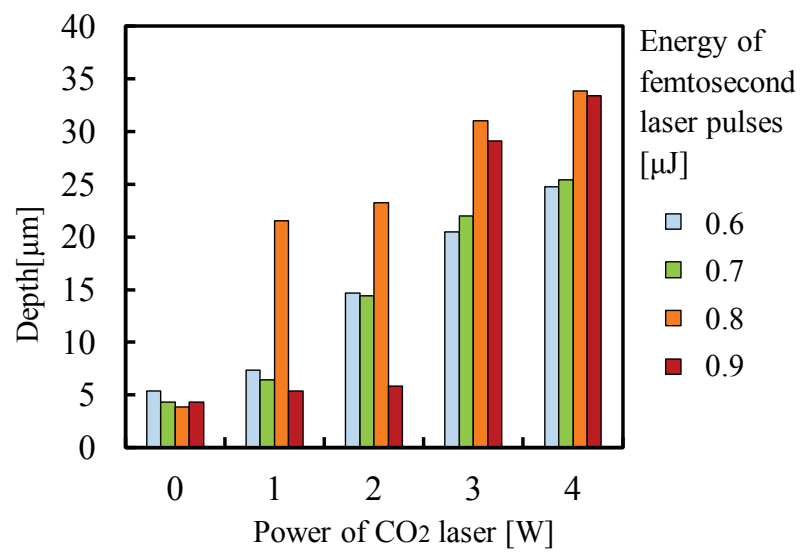

Fig. 7 Relationship between the depth of the morphology and the power of $\mathrm{CO}_{2}$ laser beam.
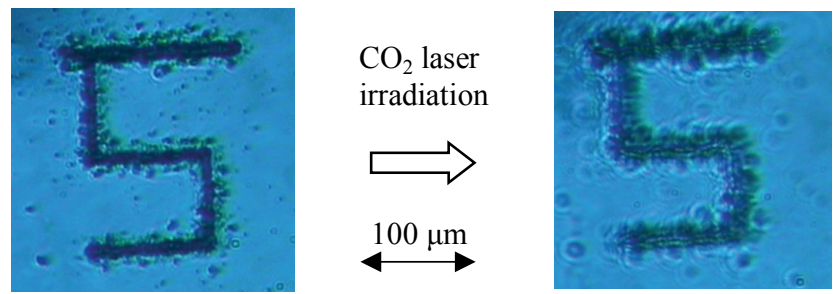

Fig. 8 Thermal effect during laser microprocessing using $\mathrm{CO}_{2}$ laser

\subsubsection{Dependence of laser-induced structural modifi- cations on scan speeds}

The purpose in this section is to clarify the dependence of laser-induced structural modifications inside BK7 glass on scan speeds by using TLAM. By translating the glass sample perpendicular to the optical axis of femtosecond laser pulses with a distance of $1 \mathrm{~mm}$, the linear structural modification regions were created. The dependence of laser-induced structural modifications on scan speeds was investigated by changing scan speed from $0.1 \mathrm{~mm} / \mathrm{s}$ to $1.0 \mathrm{~mm} / \mathrm{s}$ at an interval of $0.1 \mathrm{~mm} / \mathrm{s}$. The output power was also changed to $1 \mathrm{~W}, 2 \mathrm{~W}$, and 3 W. Table 5 shows the laser-induced morphology in each condition at intervals of $0.2 \mathrm{~mm} / \mathrm{s}$. In Table 5 , open circle $(\circ)$ and cross mark $(\times)$ show internal processing and surface ablation, respectively. From Table 5 the laser-induced morphologies are internal processing in either case of the $\mathrm{CO}_{2}$ laser output is $2 \mathrm{~W}$ and scan speed is less than $0.6 \mathrm{~mm} / \mathrm{s}$ or $\mathrm{CO}_{2}$ laser output is $3 \mathrm{~W}$. The laser-induced morphologies are surface ablation in either case of the $\mathrm{CO}_{2}$ laser output is $2 \mathrm{~W}$ and scan speed is more than $0.8 \mathrm{~mm} / \mathrm{s}$ or $\mathrm{CO}_{2}$ laser output is $1 \mathrm{~W}$. From these results we assume that it is an internal processing when the thermal effects are given enough.

Table 4 The laser-induced morphology in each condition. Open circle $(\circ)$ and cross mark $(\times)$ show internal processing and surface ablation, respectively.

\begin{tabular}{cccccc}
\hline Scan speed $[\mathrm{mm} / \mathrm{s}]$ & & & & & \\
$\mathrm{CO}_{2}$ laser power $[\mathrm{W}]$ & 0.2 & 0.4 & 0.6 & 0.8 & 1.0 \\
\hline 1 & & & & & \\
2 & $\times$ & $\times$ & $\times$ & $\times$ & $\times$ \\
3 & $\circ$ & $\circ$ & $\circ$ & $\times$ & $\times$ \\
\hline
\end{tabular}

\subsection{Internal processing}

\subsubsection{The effect of $\mathrm{CO}_{2}$ laser beam for the laser-} induced morphology inside glass

In order to reveal the effect by $\mathrm{CO}_{2}$ laser beam in TLAM, we demonstrated the laser microprocessing of BK7 glass using FSLAM or TLAM. The pulse energy of femtosecond laser was set to $1.3 \mu \mathrm{J}$. The output power of $\mathrm{CO}_{2}$ laser power was set to $5 \mathrm{~W}$. Irradiation time of femtosecond laser was $0.05 \mathrm{sec}$ and that of $\mathrm{CO}_{2}$ laser was $5 \mathrm{sec}$. Figures 9(a) and (b) show optical images of processed material using FSLAM and TLAM, respectively. From Figs. 9(a) and (b), both of FSLAM and TLAM resulted in refractive index change inside glass. The diameter of the refractive index change region using TLAM is twice that using FSLAM.

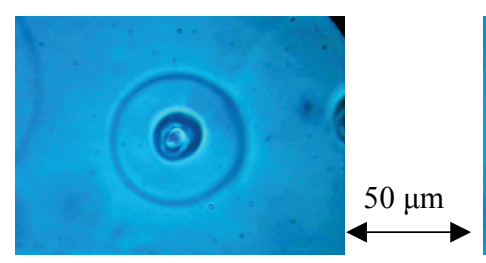

(a) FSLAM

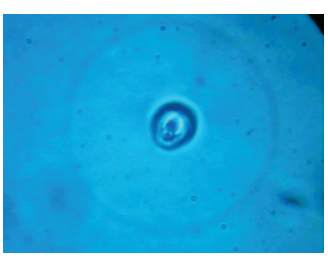

(b) TLAM
Fig. 9 Optical images of processed material using (a) FSLAM or (b) femtosecond and $\mathrm{CO}_{2}$ laser systems ( TLAM ).

\subsubsection{Dependence of diameter and magnification of the laser-induced structural modifications on output power of $\mathrm{CO}_{2}$ laser beam and energy of femtosecond laser pulses \\ We investigated the diameter and magnification of the} structural modifications by changing the output power of $\mathrm{CO}_{2}$ laser beam and/or energy of femtosecond laser pulses. The pulse energy of femtosecond laser was changed from $0.8 \mu \mathrm{J}$ to $1.3 \mu \mathrm{J}$ at an interval of $0.1 \mu \mathrm{J}$. The output power of $\mathrm{CO}_{2}$ laser beam was also changed from $0 \mathrm{~W}$ to $5 \mathrm{~W}$ at an interval of $1 \mathrm{~W}$. Figure 10 shows the examples of the change in the diameter of the refractive index change region when the pulse energy of femtosecond laser was set to $1.3 \mu \mathrm{J}$. Figure 11 shows the diameter of the refractive index change region in each condition. Figure 12 shows the magnification of the diameter of the refractive index change region using TLAM compared with that using FSLAM. Magnification is calculated from formula (1). In Fig. 12 the increasing rate of magnification in the higher energy of femtosecond laser (e.g., $1.2 \mu \mathrm{J}$ and $1.3 \mu \mathrm{J}$ ) is bigger than that in the smaller that (e.g., $0.8 \mu \mathrm{J}$ and $0.9 \mu \mathrm{J}$ ). From Fig. 11 and Fig. 12, we confirmed that TLAM increased heat-affected zone in the unit time than that using FSLAM. The processed diameter using TLAM is twice the size of the diameter using FSLAM.

$$
\text { Magnification }=\frac{\text { The diameter using TLAM }}{\text { The diameter using SFLAM }}
$$




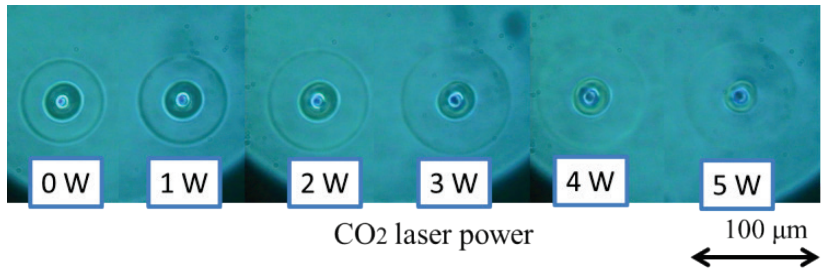

Fig. 10 Dependence of the diameter of refractive index change region on $\mathrm{CO}_{2}$ laser power.

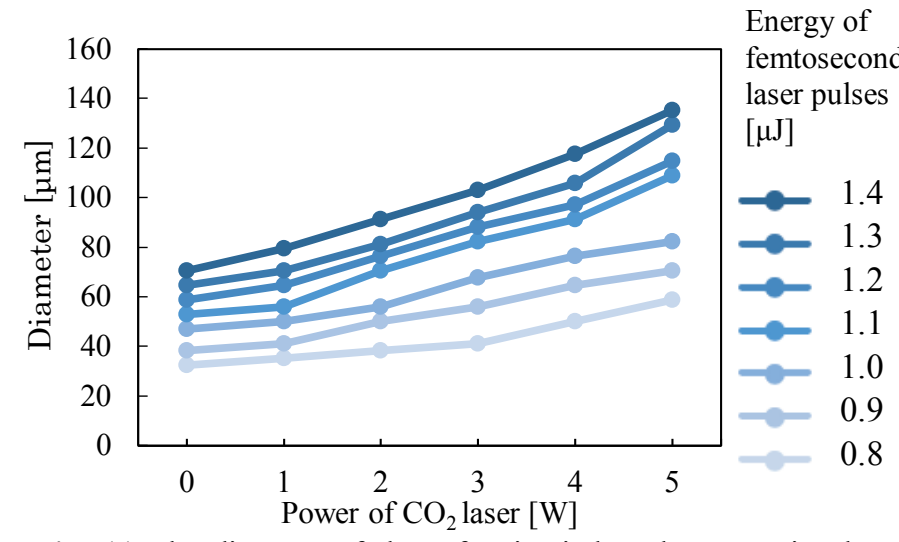

Fig. 11 The diameter of the refractive-index change region by changing the output power of $\mathrm{CO}_{2}$ laser beam and/or energy of femtosecond laser pulses.

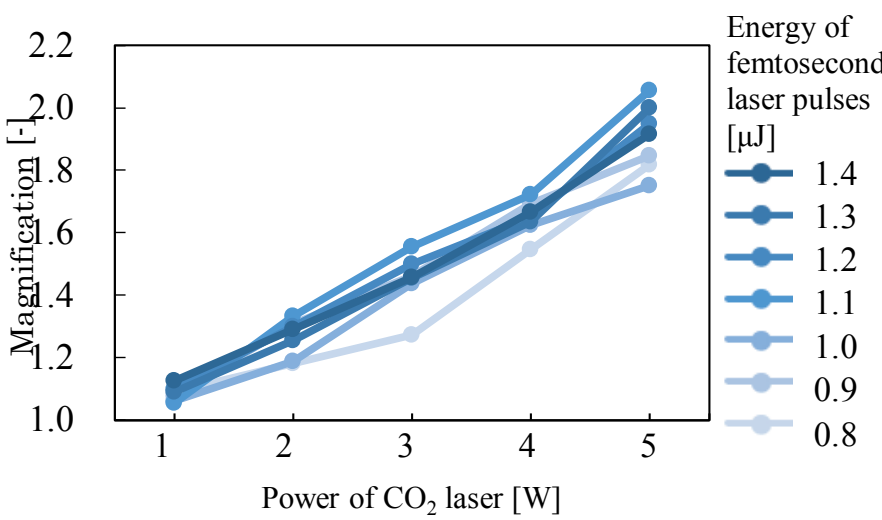

Fig. 12 The magnification of the diameter of the refractive-index change region by changing the output power of $\mathrm{CO}_{2}$ laser beam and/or energy of femtosecond laser pulses.

\section{Conclusion}

We have investigated the laser-induced structural modifications in a BK7 glass sample (OHARA, S-BSL7) using a high-repetition-rate femtosecond fiber laser and a $\mathrm{CO}_{2}$ laser system. For the depth dependence of laser-induced morphology on output power of $\mathrm{CO}_{2}$ laser beam and/or energy of femtosecond laser pulses, the depth is increased with an increase in the power of $\mathrm{CO}_{2}$ laser beam and the energy of femtosecond laser pulses except the region of surface ablation. This results show that the laser microprocessing using femtosecond and $\mathrm{CO}_{2}$ laser systems is possible to change the laser-induced morphology of BK7 glass. Also we have investigated internal processing in BK7 glass with the 2wavelengths laser microprocessing(TLAM) using femtosecond laser system and $\mathrm{CO}_{2}$ laser system. The diameter of the refractive-index change region changed by changing the out put power of $\mathrm{CO}_{2}$ laser beam and/or energy of femtosecond laser pulses. The diameter of the refractive index change region using TLAM is approximately twice the size of the diameter using single femtosecond laser microprocessing (SFLAM). The increase of the diameter of structural modifications expands the possibilities of the application to the three dimensional microfabrication inside the transparent material.

\section{References}

[1] K. Itoh, W. Watanabe, S. Nolte, and C. B. Schaffer, "Ultrafast processes for bulk modification of transparent materials," MRS Bulletin, 31 (2006) 620-625.

[2] M. Shimizu1, M. Sakakura, M. Ohnishi, Y.Shimotsuma, T. Nakaya, K. Miura1, and Kazuyuki Hirao, "Mechanism of heat-modification inside a glass after irradiation with high-repetition rate femtosecond laser pulses,"

[3] K. M. Davis, K. Miura, N. Sugimoto, and K. Hirao, "Writing waveguides in glass with a femtosecond laser," Opt. Lett., 21 (1996) 1729-1731.

[4] K. Yamada, W. Watanabe, T. Toma, K. Itoh, and J. Nishii, "In situ observation of photoinduced refractive-index changes in filaments formed in glasses by femtosecond laser pulses," Opt. Lett., 26 (2001) 19-21.

[5] D. Homoelle, S. Wielandy, A. L. Gaeta, N. F. Borrelli, and C. Smith, "Infrared photosensitivity in silica glasses exposed to femtosecond laser pulses," Opt. Lett., 24, (1999) 1311-1313.

[6] K. Yamada, W. Watanabe, Y. Li, K. Itoh, and J. Nishii, "Multilevel phase-type diffractive lenses in silica glass induced by filamentation of femtosecond laser pulses," Opt. Lett., 29 (2004) 1846-1848.

[7] L. Sudrie, M. Franco, B. Prade, and A. Mysyrowicz, "Writing of permanent birefringent microlayers in bulk fused silica with femtosecond laser pulses," Opt. Commun., 171 (1999) 279-284.

[8] K. Yamada, W. Watanabe, K. Kintaka, J. Nishii, and K. Itoh, "Volume grating induced by a self-trapped long filament of femtosecond laser pulses in Silica Glass" Jpn. J. Appl. Phys., 42 (2003) 6916-6919.

[9] K. Kawamura, N. Sarukura, M. Hirano, and H. Hosono, "Holographic encoding of fine-pitched micrograting structures in amorphous $\mathrm{SiO}_{2}$ thin films on silicon by a single femtosecond laser pulse," Appl. Phys. Lett., 78 (2001) 1038-1341.

[10] Y. Li, W. Watanabe, K. Yamada, T. Shinagawa, K. Itoh, J. Nishii, and Y. Jiang, "Holographic fabrication of multiple layers of grating inside soda-lime glass with femtosecond laser pulses," Appl. Phys. Lett., 80 (2002) 1508-1511.

[11] J. P. Gordon, R. C. C. Leite, R. S. Moore, S. P. S. Porto, and J. R. Whinnery, "Long-transient effects in lasers with inserted liquid samples,” J. Appl. Phys., 36 (1965) 3-8.

[12] Terazima, M., Horiguchi, M., and Azumi, T., Anal. Chem.61, (1989) 883-888.

[13] T.M.A., Rasheed, K.P.B., Moosad, V.P.N., Nampoori and K.Sathianandan Spectro Chim. Acta.A, 43, (1973) 72 
[14] P.E.Dyer, I.Waldeck, G.C. Roberts, J.Phys. D.Appl, Phys. 30, (1997) 19.

[15] A. Brodeur and S.L. Chin, "Ultrafast white-light continuum generation and self-focusing in transparent condensed media," JOSA B 16 (1999) 637-650.

[16] http://www.ohara-gmbh.com/e/katalog/d_sbsl7_e.html

(Received: July 25, 2013, Accepted: May 14, 2014) 